\title{
Robust Energy-Efficient Precoding Optimization for Dual-Polarized Multiuser MIMO Downlink
}

\author{
Shiqi Gong ${ }^{\dagger}$, Chengwen Xing ${ }^{\dagger}$, Sheng Chen ${ }^{\ddagger}$, Fellow, IEEE, Nan Yang* and Yiqing Zhou** \\ $\dagger$ School of Information and Electronics, Beijing Institute of Technology, Beijing 100081, China \\ E-mail: gsqyx@163.com and xingchengwen@gmail.com \\ ${ }^{\ddagger}$ Electronics and Computer Science, University of Southampton, Southampton SO17 1BJ, U.K. \\ E-mail:sqc@ecs.soton.ac.uk \\ ${ }^{*}$ Research School of Engineering, The Australian National University, Canberra, ACT 2601, Australia \\ E-mail: nan.yang@anu.edu.au). \\ ** Institute of Computing Technology, Chinese Academy of Sciences, Beijing, 100190, China \\ E-mail: zhouyiqing@ict.ac.cn
}

\begin{abstract}
In this paper, we propose the robust fairness-based energy efficiency (EE) optimization of the dual-polarized multiuser multiple-input multiple-output (MIMO) downlink. Exploiting the special dual-polarized antenna structure, the polarizationbased subgrouping technique is adopted which enjoys low implementation complexity, low feedback overhead, and good performance. Based on this, the proposed robust fairness-based EE precoding design aims at maximizing the minimum EE, i.e., the worst-case EE, achieved among all users with the norm bounded channel errors. Further, the formulated nonconvex EE optimization problem is transformed into a series of standard semidefinite programming (SDP) problems, which can be effectively solved by the convex optimization technique. Simulation results demonstrate the robust $\mathrm{EE}$ performance advantages of the proposed polarization-based subgrouping precoding scheme over the existing schemes.

Index Terms-Dual-polarized MIMO downlink, polarizationbased subgrouping, energy efficiency, robust optimization
\end{abstract}

\section{INTRODUCTION}

Multiple-input multiple-output (MIMO), characterised by its spatial multiplexing gain and diversity gain, is a highly successful wireless technology that offers many meritorious achievements [1]. However, space limitations usually introduce correlations between adjacent antennas, which degrades the achievable performance of MIMO systems [2]. To address this issue, a space and cost effective solution is to deploy dual-polarized antenna arrays at both communication terminals. With the dual-polarized antenna array structure, multiple antennas are grouped in pairs with two orthogonally polarized antenna elements. Moreover, to further reduce implementation complexity and feedback overhead, a polarization based subgrouping technique was proposed for the dual-polarized MIMO system in [3].

In future wireless communication systems, facing with the exponential growth of multimedia traffics and the limited energy supply for mobile devices, there is an urgent need to improve the energy efficiency (EE) of wireless communication systems [4]. In particular, EE has been widely accepted as an important performance metric for beamforming and precoding optimization, and there already exists a rich body of literature addressing this issue. Generally, the perfect CSI is assumed to be available in these literature. Unfortunately, this assumption is difficult to realize in real systems because of many practical factors, e.g., feedback errors. As a result, abundant works aiming to improve the robust EE performance of wireless communication systems spring up [5]. It is noted that most of these work concentrate on the statistical EE robustness or worst-case EE robustness.

However, to the best knowledge of the authors, the energyefficient multiuser precoding design for the dual-polarized MIMO downlink with imperfect CSI has not been carried out in the existing literature. This motivates us to take a further step to investigate the robust $\mathrm{EE}$ performance of the dual-polarized multiuser MIMO downlink. Specifically, in our work, the polarization based subgrouping technique is applied to realize the robust fairness-based EE optimization for the dual-polarized multiuser MIMO downlink, which can further reduce the system CSI feedback overhead and implementation complexity. Based on this, the linear precoding matrix is optimized by exploiting the equivalent relationship between the signal-to-interference-plus-noise ratio (SINR) and the mean square error (MSE) [6], the Dinkeblach's method [7] and the sign-definiteness lemma [8]. Finally, the corresponding nonconvex EE optimization problem is solved by a series of standard semidefinite programming (SDP) optimization.

\section{DuAl-Polarized Downlink Multiuser MiMO SYSTEM}

We study the multiuser MIMO downlink communication scenario depicted in Fig. 1, in which the base station (BS) equipped with $N_{t}$ polarized antennas serves $M$ mobile users (MUs) each equipped with a single horizontally or vertically polarized antenna. Here, $N_{t}$ is set to be an even number, thus the BS is equipped with $N_{t} / 2$ pairs of co-located horizontally and vertically polarized antennas [3]. Besides, the serving $M$ MUs are clustered into $K$ groups each composed of $N_{u}=\frac{M}{K}$ MUs. Generally, $N_{t}>N_{u}$ is assumed and each MU group 


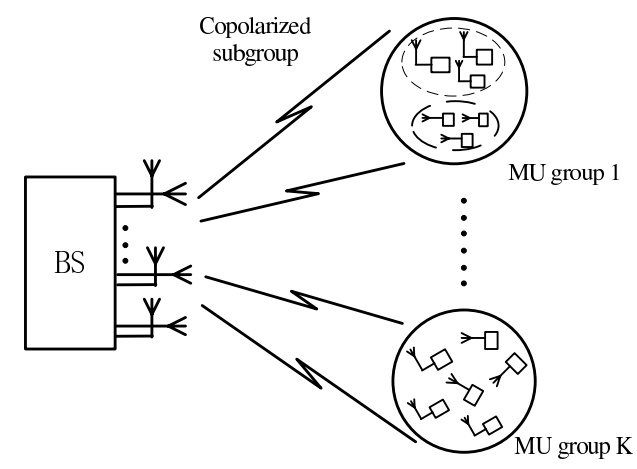

Fig. 1. The downlink of dual-polarized MU-MIMO system [3].

consists of the same number of vertically and horizontally polarized MUs. Then the received signal $\boldsymbol{y}_{k} \in \mathbb{C}^{N_{u}}$ of the $k$ th MU group can be expressed as

$$
\begin{aligned}
\boldsymbol{y}_{k} & =\left[\begin{array}{c}
\boldsymbol{y}_{k}^{v} \\
\boldsymbol{y}_{k}^{h}
\end{array}\right]=\boldsymbol{H}_{k}^{\mathrm{H}} \sum_{j \in \mathcal{K}} \boldsymbol{P}_{j} \boldsymbol{s}_{j}+\boldsymbol{n}_{k}, \\
& =\left[\begin{array}{cc}
\left(\boldsymbol{H}_{k}^{v v}\right)^{\mathrm{H}} & \left(\boldsymbol{H}_{k}^{v h}\right)^{\mathrm{H}} \\
\left(\boldsymbol{H}_{k}^{h v}\right)^{\mathrm{H}} & \left(\boldsymbol{H}_{k}^{h h}\right)^{\mathrm{H}}
\end{array}\right] \sum_{j \in \mathcal{K}} \boldsymbol{P}_{j}\left[\begin{array}{c}
\boldsymbol{s}_{j}^{v} \\
\boldsymbol{s}_{j}^{h}
\end{array}\right]+\left[\begin{array}{c}
\boldsymbol{n}_{k}^{v} \\
\boldsymbol{n}_{k}^{h}
\end{array}\right],
\end{aligned}
$$

where $\boldsymbol{y}_{k}^{p} \in \mathbb{C}^{\frac{N_{u}}{2}}, \forall p \in\{h, v\}$ and $\mathcal{K}=\{1,2, \cdots, K\}$ denotes the set of the $K$ MU groups, $\boldsymbol{s}_{j}=\left[\left(\boldsymbol{s}_{j}^{v}\right)^{\mathrm{T}},\left(\boldsymbol{s}_{j}^{h}\right)^{\mathrm{T}}\right]^{\mathrm{T}} \in \mathbb{C}^{N_{u}}$ is the transmitted symbol vector to the $j$ th MU group with $\mathbb{E}\left\{\boldsymbol{s}_{j} \boldsymbol{s}_{j}^{\mathrm{H}}\right\}=\boldsymbol{I}_{N_{u}}$. Besides, $\boldsymbol{P}_{j}=\left[\begin{array}{ll}\widetilde{\boldsymbol{P}}_{j}^{v} & \widetilde{\boldsymbol{P}}_{j}^{h}\end{array}\right]$ with $\widetilde{\boldsymbol{P}}_{j}^{p} \in$ $\mathbb{C}^{N_{t} \times \frac{N_{u}}{2}}, \forall p \in\{v, h\}$ is the precoding matrix for the $j$ th MU group with $p$ polarization, and $\boldsymbol{H}_{k} \in \mathbb{C}^{N_{t} \times N_{u}}$ represents the channel matrix linking the BS to $N_{u}$ MUs of the $k$ th group, where $\boldsymbol{H}_{k}^{m n} \in \mathbb{C}^{\frac{N_{t}}{2} \times \frac{N_{u}}{2}}, \forall m, n \in\{h, v\}$ is the channel matrix between the $n$-polarized BS antennas and the $m$-polarized MUs in the $k$ th group. $\boldsymbol{n}_{k}=\left[\left(\boldsymbol{n}_{k}^{v}\right)^{\mathrm{T}},\left(\boldsymbol{n}_{k}^{h}\right)^{\mathrm{T}}\right]^{\mathrm{T}} \in \mathbb{C}^{N_{u}}$ is the corresponding additive white Gaussian noise (AWGN) vector, whose elements obey the Gaussian distribution with zero mean and power $\sigma_{n}^{2}$. Particularly, according to [3], the dual-polarized MIMO channel is modeled as

$$
\boldsymbol{H}_{k}=\left(\left[\begin{array}{cc}
1 & \gamma_{p} \\
\gamma_{p} & 1
\end{array}\right] \otimes\left(\boldsymbol{U}_{k} \boldsymbol{\Lambda}_{k}^{\frac{1}{2}}\right)\right)\left(\boldsymbol{G}_{k} \odot\left(\boldsymbol{X} \otimes \mathbf{1}_{r_{k} \times \frac{N u}{2}}\right)\right),
$$

where $\otimes$ and $\odot$ denote the Kronecker product and Hadamard product, respectively, while $\operatorname{Tr}(\cdot)$ denotes the matrix trace operator. Besides, $\gamma_{p} \approx 0$ reflects the correlation between the horizontally and vertically polarized antennas. $\boldsymbol{G}_{k} \in \mathbb{C}^{2 r_{k} \times N_{u}}$ and $\boldsymbol{X} \in \mathbb{R}^{2 \times 2}$ are defined respectively as

$$
\boldsymbol{G}_{k}=\left[\begin{array}{ll}
\boldsymbol{G}_{k}^{v v} & \boldsymbol{G}_{k}^{h v} \\
\boldsymbol{G}_{k}^{v h} & \boldsymbol{G}_{k}^{h h}
\end{array}\right], \boldsymbol{X}=\left[\begin{array}{cc}
1 & \sqrt{\chi} \\
\sqrt{\chi} & 1
\end{array}\right] .
$$

Here $\boldsymbol{G}_{k}$ is the short-term channel fading matrix with $\boldsymbol{G}_{k}^{m n} \in$ $\mathbb{C}^{r_{k} \times \frac{N_{u}}{2}}, \forall m, n \in\{h, v\}$, and $\boldsymbol{X}$ with $0 \leq \chi \leq 1$ indicates the channel ability to separate vertical and horizontal polarizations [9]. In our work, the ideal case of $\chi \approx 0$ is considered, which means the orthogonally polarized signals are perfectly distinguished from each other. Generally, the feedback of $\boldsymbol{G}_{k}$ to the BS is imperfect due to many factors, such as quantization and feedback errors. Therefore, the short-term
CSI can be expressed as

$$
\boldsymbol{G}_{k}=\widehat{\boldsymbol{G}}_{k}+\boldsymbol{\Delta}_{k},\left\|\boldsymbol{\Delta}_{k}\right\|_{F} \leq \sigma_{k},
$$

$\forall k \in \mathcal{K}$, where $\widehat{\boldsymbol{G}}_{k}$ denotes the estimated nominal channel, and $\boldsymbol{\Delta}_{k}$ is the norm bounded CSI error by $\sigma_{k}$. In our work, the ideal case $\chi \approx 0$ is considered, which means the polarized signals are perfectly orthogonal, and the interference signals from the cross-polarized channels can be perfectly canceled out. Specifically, when $\chi \approx 0$, the channel matrix $\boldsymbol{H}_{k}$ is block diagonal, i.e., $\boldsymbol{H}_{k} \approx\left[\begin{array}{cc}\boldsymbol{H}_{k}^{v v} & \mathbf{0} \\ \mathbf{0} & \boldsymbol{H}_{k}^{h h}\end{array}\right]$. Moreover, with the subgrouping technique of separating the vertically and horizontally polarized MUs into two subgroups, the linear precoding matrix $\boldsymbol{P}_{k} \in \mathbb{C}^{N_{t} \times N_{u}}$ can be re-expressed as $\boldsymbol{P}_{k}=\left[\begin{array}{cc}\boldsymbol{P}_{k}^{v} & \mathbf{0} \\ \mathbf{0} & \boldsymbol{P}_{k}^{h}\end{array}\right]$, where $\boldsymbol{P}_{k}^{p} \in \mathbb{C}^{\frac{N_{t}}{2} \times \frac{N_{u}}{2}}$ for $p \in\{v, h\}$ denotes the $p$-polarization related precoding matrix. Therefore, the design of $\boldsymbol{P}_{k}, \forall k \in \mathcal{K}$, is transformed into the designs of the polarization related $\boldsymbol{P}_{k}^{p}$ for $p \in\{h, v\}$ utilizing the reduced-dimensional instantaneous CSI.

Further, based on (1), the received signal of each MU can be expressed as

$$
\begin{gathered}
y_{k, i}^{p} \boldsymbol{h}_{k k, i}^{p} \boldsymbol{p}_{k}^{p, i} s_{k, i}^{p}+\sum_{\substack{l \in\left\{1, \cdots, \frac{N_{u}}{2}\right\} \\
l \neq i}} \boldsymbol{h}_{k k, i}^{p} \boldsymbol{p}_{k}^{p, l} s_{k, l}^{p}+\sum_{j \in \mathcal{K} \backslash k} \boldsymbol{h}_{k j, i}^{p} \boldsymbol{P}_{j}^{p} \boldsymbol{s}_{j}^{p}+n_{k, i}^{p} \\
\forall i \in\left\{1,2, \cdots, \frac{N_{u}}{2}\right\}, p \in\{h, v\}, k \in \mathcal{K}
\end{gathered}
$$

where $\boldsymbol{h}_{k j, i}^{p}$ denotes the $i$ th row of the virtual channel $\left(\boldsymbol{H}_{k}^{p p}\right)^{\mathrm{H}}$ and $\boldsymbol{p}_{k}^{p, i}$ denotes the $i$ th column of the precoding matrix $\boldsymbol{P}_{k}^{p}$, for $p \in\{h, v\}$. Hence, the achievable user rate is given by

$$
R_{k, i}^{p}=\log \left(1+\operatorname{SINR}_{k, i}^{p}\right),
$$

with the corresponding SINR given by

$\operatorname{SINR}_{k, i}^{p}=\frac{\left|\boldsymbol{h}_{k k, i}^{p} \boldsymbol{p}_{k}^{p, i}\right|^{2}}{\sum_{l \in\left\{1, \cdots, \frac{N_{u}}{2}\right\}, l \neq i}\left|\boldsymbol{h}_{k k, i}^{p} \boldsymbol{p}_{k}^{p, l}\right|^{2}+\sum_{j \in \mathcal{K} \backslash k}\left\|\boldsymbol{h}_{k j, i}^{p} \boldsymbol{P}_{j}^{p}\right\|^{2}+\sigma_{n}^{2}}$,

and the related user power consumption is re-expressed as $\mathrm{PW}_{k, i}^{p}=\operatorname{Tr}\left(\boldsymbol{p}_{k}^{p, i}\left(\boldsymbol{p}_{k}^{p, i}\right)^{\mathrm{H}}\right)+P_{c}=\left\|\boldsymbol{p}_{k}^{p, i}\right\|^{2}+P_{c}$. Thus the achievable EE of the $i$ th MU with $p$-polarization in the $k$ th group is reformulated as

$$
\mathrm{EE}_{k, i}^{p}=\frac{R_{k, i}^{p}}{\mathrm{PW}_{k, i}^{p}}
$$

We now apply the imperfect instantaneous CSI model with the norm bounded errors in (4) to the virtual channel $\left(\boldsymbol{H}_{k}^{p p}\right)^{\mathrm{H}}$ to obtain

$$
\begin{aligned}
\boldsymbol{h}_{k j, i}^{p} & =\widehat{\boldsymbol{h}}_{k j, i}^{p}+\boldsymbol{\Delta}_{k j, i}^{p},\left\|\boldsymbol{\Delta}_{k j, i}^{p}\right\| \leq \tau_{k j, i}^{p} \\
& p \in\{h, v\}, \quad i \in\left\{1,2, \cdots, \frac{N_{u}}{2}\right\}, k, j \in \mathcal{K},
\end{aligned}
$$


where $\widehat{\boldsymbol{h}}_{k j, i}^{p}$ and $\boldsymbol{\Delta}_{k j, i}^{p}$ denote the estimated nominal channel and the corresponding CSI error for $\boldsymbol{h}_{k j, i}^{p} \in \mathbb{C}^{1 \times \frac{L}{2}}$, respectively, and the error threshold $\tau_{k j, i}^{p}$ satisfies $\tau_{k j, i}^{p}=\sqrt{\frac{\operatorname{Tr}\left(\boldsymbol{R}_{k}\right)}{2 r_{k} N_{u}}} \sigma_{k}$. Based on (8) and (9), the robust fairness-based EE optimization problem is formulated as

$$
\begin{array}{r}
\max _{\boldsymbol{P}_{k}^{p}, k \in \mathcal{K}, p \in\{h, v\}} \min _{\forall i \in\left\{1, \cdots, \frac{N u}{2}\right\}, k \in \mathcal{K}, p \in\{h, v\}} \min _{\boldsymbol{\Delta}_{\mathcal{K}}} \mathrm{EE}_{k, i}^{p}, \\
\text { s.t. } \sum_{k \in \mathcal{K}} \sum_{p \in\{h, v\}} \operatorname{Tr}\left(\boldsymbol{P}_{k}^{p}\left(\boldsymbol{P}_{k}^{p}\right)^{\mathrm{H}}\right) \leq P_{\max },
\end{array}
$$

where $\boldsymbol{\Delta}_{\mathcal{K}}=\left\{\boldsymbol{\Delta}_{k j, i}^{p}: \forall i, k, j, p\right\}$ denotes the set of all CSI uncertainties in the dual-polarized MIMO downlink. Clearly, the problem (10) indicates that the minimum EE among all MUs is maximized considering the imperfect CSI. However, the non-convex fractional problem (10) is difficult to solve due to the intractable objective function. To address this issue, we utilize the Dinkeblach's method [7] and propose an iterative optimization algorithm in the following Section.

\section{Proposed Iterative EE Optimization}

In order to tackle the optimization problem (10) effectively, we first transform (10) into the following equivalent problem utilizing the Dinkeblach's method [7], which leads to

$$
\min _{\substack{\forall i \in\left\{1, \cdots, \frac{N u}{u}\right\} \\ k \in \mathcal{K}, p \in\{h, v\}, \eta}} G_{k, i}^{p}(\eta), \text { s.t. } \sum_{k \in \mathcal{K}} \sum_{p \in\{h, v\}}\left\|\boldsymbol{P}_{k}^{p}\right\|_{F}^{2} \leq P_{\max },
$$

where the objective function $G_{k, i}^{p}(\eta)$ is defined as

$$
G_{k, i}^{p}(\eta)=\max _{\boldsymbol{P}_{k}^{p}} R_{k, i}^{p \star}-\eta \mathrm{PW}_{k, i}^{p},
$$

which is monotonically decreasing with respect to the auxiliary variable $\eta$. Following the similar logic given in [5], it is concluded that the optimal precoding matrix of problem (11) is obtained by satisfying $\min _{\forall i k, p, \eta} G_{k, i}^{p}(\eta)=0$. Furthermore, it can be proved that $\eta$ falls in the following range $\eta \in\left[0, \frac{R_{k \max }^{p \max }}{\mathrm{PW}_{k, i}^{p \min }}\right]$, where $\mathrm{PW}_{k, i}^{p \min }=P_{c}$ and $R_{k, i}^{p \max }=\log \left(1+P_{\max }\left\|\widehat{\boldsymbol{h}}_{k k, i}^{p}\right\|^{2}\right)$. Refer to [7], the optimal $\eta$ can be found via a one-dimensional search. However, even with $\eta$ fixed, the optimization (11) is nonconvex due to the nonlinear optimization objective. To overcome this difficulty, $G_{k, i}^{p}(\eta)$ is further reformulated by exploiting the equivalent relationship between the achievable SINR and the MSE of the desired signal [6]. More specifically, let $f_{k, i}^{p}$ denote the equalizer for the $i$ th MU with $p$-polarization in the $k$ th group. Then the MSE of the desired signal $s_{k}^{p, i}$ is given by

$$
\begin{aligned}
& \operatorname{MSE}_{k, i}^{p}=\mathbb{E}\left\{\left|s_{k, i}^{p}-f_{k, i}^{p} y_{k, i}^{p}\right|^{2}\right\} \\
& =\left\|f_{k, i}^{p} \boldsymbol{h}_{k k, i}^{p} \boldsymbol{P}_{k}^{p}-\boldsymbol{e}_{i}\right\|^{2}+\sum_{j \in \mathcal{K} \backslash k}\left\|f_{k, i}^{p} \boldsymbol{h}_{k j, i}^{p} \boldsymbol{P}_{j}^{p}\right\|^{2}+\sigma_{n}^{2}\left|f_{k, i}^{p}\right|^{2},
\end{aligned}
$$

where $\boldsymbol{e}_{i} \in \mathbb{R}^{1 \times \frac{N u}{2}}$ is a vector whose $i$ th element is 1 and the rest elements are zero. Particularly, for the imperfect dualpolarized MIMO downlink, the relationship between SINR and MSE is $\frac{1}{1+\min _{\mathcal{K}} \operatorname{SINR}_{k, i}^{p}}=\max _{\boldsymbol{\Delta}_{\mathcal{K}}} \min _{k, i}^{p} \operatorname{MSE}_{k, i}^{p}$. Then the formulation (12) becomes

$$
\begin{aligned}
G_{k, i}^{p}(\eta) & =-\min _{\boldsymbol{P}_{k}^{p}}\left(\log \left(\max _{\boldsymbol{\Delta}_{\mathcal{K}}} \min _{f_{k, i}^{p}} \operatorname{MSE}_{k, i}^{p}\right)+\eta\left\|\boldsymbol{p}_{k}^{p, i}\right\|^{2}+\eta P_{c}\right) \\
& =-\min _{\boldsymbol{P}_{k}^{p}, f_{k, i}^{p}}\left(\max _{\boldsymbol{\Delta}_{\mathcal{K}}} \log \left(\mathrm{MSE}_{k, i}^{p}\right)+\eta\left\|\boldsymbol{p}_{k}^{p, i}\right\|^{2}+\eta P_{c}\right) \cdot(14)
\end{aligned}
$$

Based on this, the optimization (11) is relaxed to the following one

$$
\begin{aligned}
& \min _{\boldsymbol{P}_{k}^{p}, f_{k, i \forall i \in\left\{1, \cdots, \frac{N_{u}}{2}\right\}}} \max _{\substack{p \\
, k \in \mathcal{K}, p \in\{h, v\}, \eta}}\left(\max _{\boldsymbol{\Delta}_{\mathcal{K}}} \log \left(\mathrm{MSE}_{k, i}^{p}\right)+\eta\left\|\boldsymbol{p}_{k}^{p, i}\right\|^{2}+\eta P_{c}\right), \\
& \text { s.t. } \sum_{k \in \mathcal{K}} \sum_{p \in\{h, v\}}\left\|\boldsymbol{P}_{k}^{p}\right\|_{F}^{2} \leq P_{\max } .
\end{aligned}
$$

Unfortunately, to tackle this nonconvex problem, some necessary mathematical transformations are needed. Specifically, we first introduce an auxiliary function $U_{k, i}^{p}\left(\alpha_{k, i}^{p}\right)$ to replace the term $\max _{\boldsymbol{\Delta}_{\mathcal{K}}} \log \left(\mathrm{MSE}_{k, i}^{p}\right)$ in the objective of (15), which is

$$
U_{k, i}^{p}\left(\alpha_{k, i}^{p}\right)=\exp \left(\alpha_{k, i}^{p}-1\right) \max _{\boldsymbol{\Delta}_{\mathcal{K}}} \mathrm{MSE}_{k, i}^{p}-\alpha_{k, i}^{p} .
$$

Based on the lagrangian method, it can easily be shown that

$$
\min _{\alpha_{k, i}^{p}} U_{k, i}^{p}\left(\alpha_{k, i}^{p}\right)=\max _{\boldsymbol{\Delta}_{\mathcal{K}}} \log \left(\mathrm{MSE}_{k, i}^{p}\right) .
$$

Besides, combining (16) with (17), the optimization problem (15) can be rewritten as

$$
\begin{aligned}
& \min _{\boldsymbol{P}_{k}^{p}, f_{k, i}^{p}, \alpha_{k, \forall i \in\{}^{p} \in\left\{1, \cdots, \frac{N_{u}}{2}\right\}} \max _{, k \in \mathcal{K}, p \in\{h, v\}, \eta}\left(U_{k, i}^{p}\left(\alpha_{k, i}^{p}\right)+\eta\left\|\boldsymbol{p}_{k}^{p, i}\right\|^{2}+\eta P_{c}\right), \\
& \text { s.t. } \sum_{k \in \mathcal{K}} \sum_{p \in\{h, v\}}\left\|\boldsymbol{P}_{k}^{p}\right\|_{F}^{2} \leq P_{\max } .
\end{aligned}
$$

Further substituting $\operatorname{MSE}_{k, i}^{p}$ in (13) into (18), we obtain the robust EE optimization problem (19) at the top of next page, where $l_{k, i}^{p}=\exp \left(\alpha_{k, i}^{p}-1\right)\left|f_{k, i}^{p}\right|^{2}, c_{k, i}^{p}=\eta P_{c}+\sigma_{n}^{2} l_{k, i}^{p}-\alpha_{k, i}^{p}$. It is worth noting that the optimization problem (19) is actually an epigraph form of the problem (18). To solve this problem effectively, we propose a suboptimal algorithm to decompose the problem (19) into a series of iterative subproblems. The detailed iterative optimization procedure for solving the problem (19) is presented as follows.

1) Optimizing $\alpha_{k, i}^{p}$ : Since the function (16) is convex with respect to $\alpha_{k, i}^{p}$ when $\boldsymbol{P}_{k}^{p}$ and $f_{k, i}^{p}$ are fixed, we can set the derivative of $U_{k, i}^{p}\left(\alpha_{k, i}^{p}\right)$ to zero to obtain the optimal value of $\alpha_{k, i}^{p}$ as $\alpha_{k, i}^{p \star}=-\max _{\boldsymbol{\Delta}_{\mathcal{K}}} \log \left(\mathrm{MSE}_{k, i}^{p}\right)+1$. In fact, by substituting $\alpha_{k, i}^{p \star}$ into (16), we obtain the minimum value of $U_{k, i}^{p}\left(\alpha_{k, i}^{p}\right)$ as $\max _{\boldsymbol{\Delta}_{\mathcal{K}}} \log \left(\mathrm{MSE}_{k, i}^{p}\right)$, which is consistent with (17). Clearly, the $\Delta_{\mathcal{K}}$
optimization $\alpha_{k, i}^{p \star}$ is a standard convex problem due to the concavity of the log function. 


$$
\begin{aligned}
& \min _{\boldsymbol{P}_{k}^{p}, f_{k, i}^{p}, \alpha_{k, i}^{p}} \gamma, \\
& \text { s.t. } \forall k, j \in \mathcal{K}, j \neq k, i \in\left\{1, \cdots, \frac{N_{u}}{2}\right\}, p \in\{h, v\}: \\
& \max _{\boldsymbol{\Delta}_{k k, i}^{p}} l_{k, i}^{p}\left\|\boldsymbol{h}_{k k, i}^{p} \boldsymbol{P}_{k}^{p}-\frac{1}{f_{k, i}^{p}} \boldsymbol{e}_{i}\right\|^{2}+\max _{\boldsymbol{\Delta}_{k j, i}^{p}} l_{k, i}^{p} \sum_{j \in \mathcal{K} \backslash k}\left\|\boldsymbol{h}_{k j, i}^{p} \boldsymbol{P}_{j}^{p}\right\|^{2}+\eta\left\|\boldsymbol{p}_{k}^{p, i}\right\|^{2}+c_{k, i}^{p} \leq \gamma, \sum_{k \in \mathcal{K}} \sum_{p \in\{h, v\}}\left\|\boldsymbol{P}_{k}^{p}\right\|_{F}^{2} \leq P_{\max },
\end{aligned}
$$

2) Optimizing $\boldsymbol{P}_{k}^{p}$ : Given $\alpha_{k, i}^{p}$ and $f_{k, i}^{p}$, the optimization (19) becomes the one that with respect to $\boldsymbol{P}_{k}^{p}$ only, i.e., $\min _{\boldsymbol{P}_{k}^{p}} \gamma$. To transform this optimization into a standard SDP problem, we introduce some auxiliary variables, i.e., $b_{k j, i}^{p}$ and $d_{k}^{p, i}$, to simplify its constraints so that the problem is reformulated as

$$
\begin{aligned}
& \min _{\boldsymbol{P}_{k}^{p}, b_{k j, i}^{p}, d_{k}^{p, i}} \gamma, \\
& \text { s.t. } \forall k, j \in \mathcal{K}, j \neq k, i \in\left\{1, \cdots, \frac{N_{u}}{2}\right\}, p \in\{h, v\}: \\
& \quad \sqrt{l_{k, i}^{p}}\left\|\boldsymbol{h}_{k k, i}^{p} \boldsymbol{P}_{k}^{p}-\frac{1}{f_{k, i}^{p}} \boldsymbol{e}_{i}\right\| \leq b_{k k, i}^{p},\left\|\boldsymbol{\Delta}_{k k, i}^{p}\right\| \leq \tau_{k k, i}^{p}, \\
& \sqrt{l_{k, i}^{p}}\left\|\boldsymbol{h}_{k j, i}^{p} \boldsymbol{P}_{j}^{p}\right\| \leq b_{k j, i}^{p},\left\|\boldsymbol{\Delta}_{k j, i}^{p}\right\| \leq \tau_{k j, i}^{p}, \\
& \sum_{j \in \mathcal{K}}\left(b_{k j, i}^{p}\right)^{2}+\left(d_{k}^{p, i}\right)^{2}+c_{k, i}^{p} \leq \gamma, \sum_{k \in \mathcal{K}} \sum_{p \in\{h, v\}}\left\|\boldsymbol{P}_{k}^{p}\right\|_{F}^{2} \leq P_{\max }, \\
& \sqrt{\eta}\left\|\boldsymbol{p}_{k}^{p, i}\right\| \leq d_{k}^{p, i},
\end{aligned}
$$

Then jointly based on the Schur complement lemma [10] and the sign-definiteness lemma [8], the first two sets of constraints in (20) are equivalent to the following finite linear matrix inequalities (LMIs)

$$
\begin{gathered}
\forall k, j \in \mathcal{K}, j \neq k, i \in\left\{1, \cdots, \frac{N_{u}}{2}\right\}, p \in\{h, v\}: \\
\boldsymbol{Z}_{k k, i}^{p} \succeq \boldsymbol{X}^{\mathrm{H}} \boldsymbol{Q}_{k k, i}^{p} \boldsymbol{Y}_{k}^{p}+\left(\boldsymbol{Y}_{k}^{p}\right)^{\mathrm{H}}\left(\boldsymbol{Q}_{k k, i}^{p}\right)^{\mathrm{H}} \boldsymbol{X}, \\
\boldsymbol{Z}_{k j, i}^{p} \succeq \boldsymbol{X}^{\mathrm{H}} \boldsymbol{Q}_{k j, i}^{p} \boldsymbol{Y}_{j}^{p}+\left(\boldsymbol{Y}_{j}^{p}\right)^{\mathrm{H}}\left(\boldsymbol{Q}_{k j, i}^{p}\right)^{\mathrm{H}} \boldsymbol{X} .
\end{gathered}
$$

where the related parameters are given in (22) at the top of next page. Then we can also reexpress the problem (20) as (23) at the top of next page. where $\boldsymbol{P}=\left[\boldsymbol{P}_{1}^{h}, \boldsymbol{P}_{1}^{v}, \cdots, \boldsymbol{P}_{K}^{h}, \boldsymbol{P}_{K}^{v}\right] \in$ $\mathbb{C}^{\frac{L}{2}} \times K N_{u}$ Clearly, the problem (23) is a standard SDP problem for the linear precoding $\boldsymbol{P}_{k}^{p}, k \in \mathcal{K}, p \in\{h, v\}$, which can be solved with an interior point method.

3) Optimizing $f_{k, i}^{p}:$ It is noted that the optimization of $f_{k, i}^{p}$ can also be cast to an SDP problem, which is similar to the the optimization of $\boldsymbol{P}_{k}^{p}$. For space limitation, the detailed derivation process is omitted here.

In a nutshell, to solve the optimization problem (19) effectively when $\eta$ is given, an iterative optimization is performed among the optimization variables $\left\{\alpha_{k, i}^{p}, \boldsymbol{P}_{k}^{p}, f_{k, i}^{p}: \forall i \in\right.$ $\left.\left\{1, \cdots, \frac{N_{u}}{2}\right\}, k \in \mathcal{K}, p \in\{h, v\}\right\}$. Furthermore, combining with the bisection method to optimize $\eta$, the optimal $\eta^{\star}$ for the original robust EE problem (11) is available.

\section{Simulation Results}

In this section, numerical simulations are conducted to assess the performance of the proposed robust dual-structured

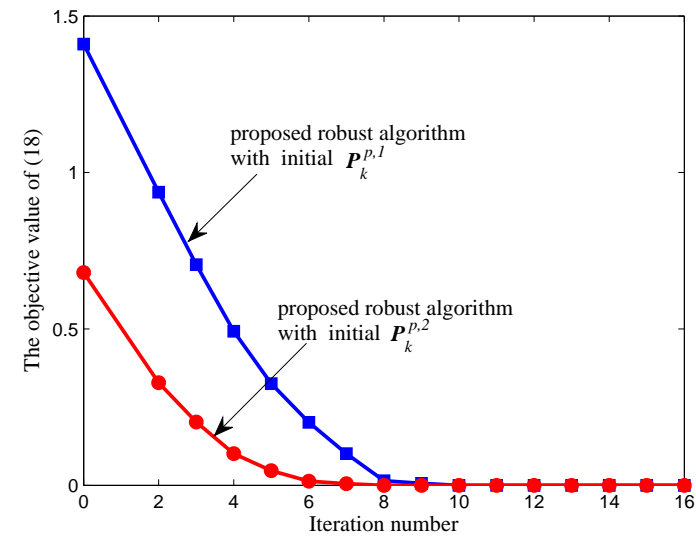

Fig. 2. Convergence of the proposed iterative algorithm for precoding optimization.

subgrouping precoding scheme. At the beginning, the following one-ring model [3] is used to calculate the spatial covariance matrix $\boldsymbol{R}_{k}$ for the MUs in the $k^{\text {th }}$ group

$$
\left[\boldsymbol{R}_{k}\right]_{m, n}=\frac{1}{2 \Delta_{k}^{a}} \int_{-\Delta_{k}^{a}}^{\Delta_{k}^{a}} e^{-j \pi \lambda_{0}^{-1} \Omega\left(\alpha+\theta_{k}\right)\left(\boldsymbol{r}_{m}-\boldsymbol{r}_{n}\right)} d \alpha
$$

where $\Delta_{k}^{a} \approx \tan ^{-1}\left(s_{k} / d_{k}\right)$ is the angular spread of the departure radio frequency (RF) signal to the $k$ th group and $\Delta_{1}^{a}=\cdots=\Delta_{K}^{a}=\frac{4 \pi}{180}$ is set. Besides, the considered system consists of one BS with $N_{t}=18$ polarized antennas and $K=3 \mathrm{MU}$ groups each with $N_{u}=4 \mathrm{MUs}$. The CSI error threshold $\sigma_{k}=0.2, \forall k \in \mathcal{K}$ is set. To solve the SDP problems (14) effectively, the famous matlab toolbox CVX [10] is utilized. Note that the non-robust algorithm is adopted as a benchmark scheme, where the EE precoder is designed by simply taking the nominal CSI as the perfect one.

In the polarization-based subgrouping precoding, the linear precoder $\boldsymbol{P}_{k}^{p}, p=\{h, v\}$ is optimized by solving (18) via the proposed iterative algorithm. Hence, we firstly investigate the convergence of the proposed algorithm in Fig. 2. From this figure, it is clear that the proposed algorithm converges after almost 10 iterations with different initial values. Here, two kinds of initials for $\boldsymbol{P}_{k}^{p}, p=\{h, v\}$ are given by

$$
\boldsymbol{P}_{k}^{p, 1}=2 \sqrt{\frac{P_{\max }}{N_{u} N_{t}}} \mathbf{1}_{\frac{N_{t}}{2} \times \frac{N_{u}}{2}}, \boldsymbol{P}_{k}^{p, 2}=\sqrt{\frac{8 P_{\max }}{N_{u} N_{t}}}\left[\mathbf{0}_{\frac{N_{t}}{2} \times \frac{N_{u}}{4}}, \mathbf{1}_{\frac{N_{t}}{2} \times \frac{N_{u}}{4}}\right]
$$




$$
\begin{aligned}
& \forall k, j \in \mathcal{K}, j \neq k, i \in\left\{1, \cdots, \frac{N_{u}}{2}\right\}, p \in\{h, v\}: \boldsymbol{X}=-\left[\begin{array}{ll}
1 & 0
\end{array}\right] \in \mathbb{R}^{1 \times\left(1 \frac{N_{u}}{2}\right)}, \boldsymbol{Y}_{k}^{p}=\left[\begin{array}{ll}
\mathbf{0} & \boldsymbol{P}_{k}^{p}
\end{array}\right] \in \mathbb{C}^{\frac{L}{2} \times\left(1 \frac{N_{u}}{2}\right)}, \boldsymbol{Q}_{k j, i}^{p}=\boldsymbol{\Delta}_{k j, i}^{p}, \\
& \boldsymbol{Z}_{k k, i}^{p}=\left[\begin{array}{cc}
\frac{b_{k k, i}^{p}}{\sqrt{l_{k, i}^{p}}} & \widehat{\boldsymbol{h}}_{k k, i}^{p} \boldsymbol{P}_{k}^{p}-\frac{1}{f_{k, i}^{p}} \boldsymbol{e}_{i} \\
\left(\boldsymbol{P}_{k}^{p}\right)^{\mathrm{H}}\left(\widehat{\boldsymbol{h}}_{k k, i}^{p}\right)^{\mathrm{H}}-\frac{1}{\left(f_{k, i}^{p}\right)^{*}} \boldsymbol{e}_{i}^{\mathrm{H}} & \frac{b_{k, i}^{p}}{\sqrt{l_{k, i}^{p}}} \boldsymbol{I}_{\frac{N u}{2}}
\end{array}\right] \in \mathbb{C}^{\left(\mathrm{H}^{+\frac{N u}{2}}\right) \times\left(\mathrm{H}^{\frac{N}{2}}\right)}, \boldsymbol{Z}_{k j, i}^{p}=\left[\begin{array}{cc}
\frac{b_{k j, i}^{p}}{\sqrt{l_{k, i}^{p}}} & \widehat{\boldsymbol{h}}_{k j, i}^{p} \boldsymbol{P}_{j}^{p} \\
\left(\boldsymbol{P}_{j}^{p}\right)^{\mathrm{H}}\left(\widehat{\boldsymbol{h}}_{k j, i}^{p}\right)^{\mathrm{H}} & \frac{b_{k j, i}^{p}}{\sqrt{l_{k, i}^{p}}} \boldsymbol{I}_{\frac{N_{u}}{2}}
\end{array}\right],
\end{aligned}
$$

$\min _{\boldsymbol{P}_{k}^{p}, b_{k j, i}^{p}, d_{k}^{p, i}} \gamma$,

s.t. $\forall k, j \in \mathcal{K}, j \neq k, i \in\left\{1, \cdots, \frac{N_{u}}{2}\right\}, p \in\{h, v\}$ :

$$
\begin{gathered}
{\left[\begin{array}{ccc}
\frac{b_{k, i}^{p}}{\sqrt{l_{k, i}^{p}} \lambda_{k k, i}^{p}} & \widehat{\boldsymbol{h}}_{k k, i}^{p} \boldsymbol{P}_{k}^{p}-\frac{1}{f_{k, i}^{p}} \boldsymbol{e}_{i} & \mathbf{0} \\
\left(\boldsymbol{P}_{k}^{p}\right)^{\mathrm{H}}\left(\widehat{\boldsymbol{h}}_{k k, i}^{p}\right)^{\mathrm{H}}-\frac{1}{\left(f_{k, i}^{p}\right)^{*}} \boldsymbol{e}_{i}^{\mathrm{H}} & \frac{b_{k k, i}^{p}}{\sqrt{l_{k, i}^{p}}} \boldsymbol{I}_{\frac{N_{u}}{2}} & -\tau_{k k, i}^{p}\left(\boldsymbol{P}_{k}^{p}\right)^{\mathrm{H}} \\
\mathbf{0} & -\tau_{k k, i}^{p} \boldsymbol{P}_{k}^{p} & \lambda_{k k, i}^{p} \boldsymbol{I}_{\frac{L}{2}}
\end{array}\right] \succeq \mathbf{0},\left[\begin{array}{ccc}
\frac{b_{k j, i}^{p}}{\sqrt{l_{k, i}^{p}}-\lambda_{k j, i}^{p}} & \widehat{\boldsymbol{h}}_{k j, i}^{p} \boldsymbol{P}_{j}^{p} & \mathbf{0} \\
\left(\boldsymbol{P}_{j}^{p}\right)^{\mathrm{H}}\left(\widehat{\boldsymbol{h}}_{k j, i}^{p}\right)^{\mathrm{H}} & \frac{b_{k j, i}^{p}}{\sqrt{l_{k, i}^{p}} \boldsymbol{I}_{\frac{N_{u}}{2}}} & -\tau_{k j, i}^{p}\left(\boldsymbol{P}_{j}^{p}\right)^{\mathrm{H}} \\
\mathbf{0} & -\tau_{k j, i}^{p} \boldsymbol{P}_{j}^{p} & \lambda_{k j, i}^{p} \boldsymbol{I}_{\frac{L}{2}}
\end{array}\right] \succeq \mathbf{0},} \\
\lambda_{k k, i}^{p}>0, \lambda_{k j, i}^{p}>0, \sqrt{\eta}\left\|\boldsymbol{p}_{k}^{p, i}\right\| \leq d_{k}^{p, i}, \sum_{j \in \mathcal{K}}\left(b_{k j, i}^{p}\right)^{2}+\left(d_{k}^{p, i}\right)^{2}+c_{k, i}^{p} \leq \gamma,\|\boldsymbol{P}\| \leq \sqrt{P_{\max }} .
\end{gathered}
$$

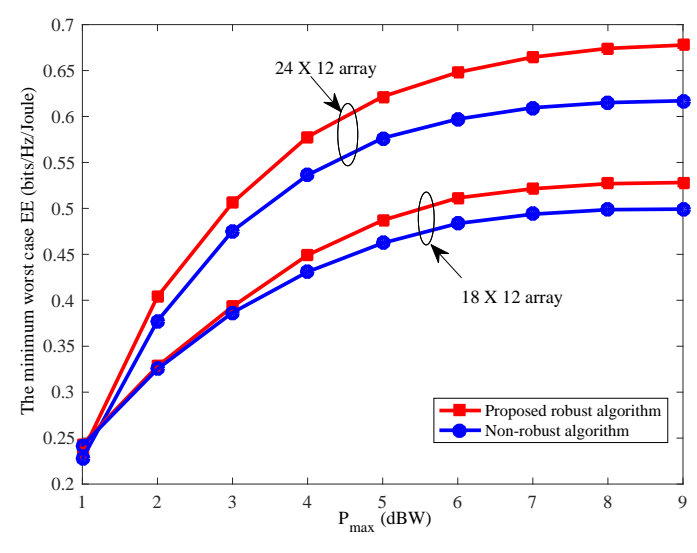

Fig. 3. The achievable minimum worst case EE versus the total transmit power $P_{\max }$ for different system scales, when $N_{t}=18 / 24, N_{u}=4, K=3$ and $P_{c}=1 \mathrm{dBW}$.

Fig. 3 illustrates the achievable minimum worst case EE among MUs versus the total transmit power $P_{\max }$ under different system scales. From this figure, it can be concluded that under the arbitrary array scale, the minimum worst case EE among MUs rises and becomes statured with the increase of $P_{\max }$ for both algorithms. In addition, compared with the non-robust scheme, the higher minimum worst case EE can be obtained using the proposed algorithm.

\section{Conclusions}

In this paper, we proposed a polarization-based subgrouping precoding scheme to realize the robust fairness-based EE optimization of the dual-polarized MU-MIMO downlink scenario, which can reduce system instantaneous CSI feedback overhead and complexity significantly. To be specific, the minimum EE among all MUs in the dual-polarized MU-MIMO downlink system was maximized considering the imperfect system CSI. Finally, numerical experiments demonstrated the good robust EE performance realized by the proposed algorithm.

\section{ACKNOWLEDGMENT}

This work was supported by the National Natural Science Foundation of China under Grant 61671058.

\section{REFERENCES}

[1] H. Weingarten, Y. Steinberg, and S. S. Shamai, "The capacity region of the Gaussian multiple-input multiple-output broadcast channel," IEEE Trans. Inf. Theory, vol. 52, no. 9, pp. 3936-3964, Sep. 2006.

[2] T. Kim, B. Clerckx, D. J. Love, and S. J. Kim, "Limited feedback beamforming systems for dual-polarized MIMO channels," IEEE Trans. Wireless Commun., vol. 9, no. 11, pp. 3425-3439, Nov. 2010.

[3] J. Park and B. Clerckx, "Multi-user linear precoding for multi-polarized massive MIMO system under imperfect CSIT," IEEE Trans. Wireless Commun., vol. 14, no. 5, pp. 2532-2547, May 2015.

[4] Z. Hasan, H. Boostanimehr, and V. K. Bhargava, "Green cellular networks: A survey, some research issues and challenges," IEEE Commun. Surveys Tuts., vol. 13, no. 4, pp. 524-540, 2011.

[5] A. Zappone, P. Cao, and E. A. Jorswieck, "Low-complexity energy efficiency optimization with statistical CSI in two-hop MIMO systems," IEEE Signal Process. Letters, vol. 21, no. 11, pp. 1398-1402, Nov. 2014

[6] S. S. Christensen, R. Argawal, and E. de Carvalho, "Weighted sumrate maximization using weighted MMSE for MIMO-BC beamforming design," IEEE Trans. Wireless Commun., vol. 7, no. 12, pp. 4792-4799, Dec. 2008.

[7] A. Aubry, V. Carotenuto, and A. De Maio, "New results on generalized fractional programming problems with Toeplitz quadratics," IEEE Signal Process. Letters, vol. 23, no. 6, pp. 848-852, Jun. 2016.

[8] E. A. Gharavol and E. G. Larsson, "The sign-definiteness lemma and its applications to robust transceiver optimization for multiuser MIMO systems," IEEE Trans. Signal Process., vol. 61, no. 2, pp. 238-252, Jan. $15,2013$.

[9] M. Coldrey, "Modeling and capacity of polarized MIMO channels," in Proc. VTC Spring 2008 (Singapore), May 11-14, 2008, pp. 440-444.

[10] S. Boyd and L. Vandenberghe, Convex Optimization. Cambridge University Press: Cambridge, UK, 2004. 